\title{
Global Prevalence of Alcohol Use and Binge Drinking During Pregnancy and Fetal Alcohol Spectrum Disorder
}

\begin{tabular}{|c|c|}
\hline Journal: & Biochemistry and Cell Biology \\
\hline Manuscript ID & bcb-2017-0077.R1 \\
\hline Manuscript Type: & Mini Review \\
\hline Date Submitted by the Author: & 11-Aug-2017 \\
\hline Complete List of Authors: & $\begin{array}{l}\text { Popova, Svetlana; Centre for Addiction and Mental Health - Russell Street } \\
\text { Site, Institute for Mental Health Policy Research; University of Toronto, } \\
\text { Dalla Lana School of Public Health; University of Toronto, Institute of } \\
\text { Medical Science; University of Toronto, Factor-Inwentash Faculty of Social } \\
\text { Work } \\
\text { Lange, Shannon; Centre for Addiction and Mental Health, Institute for } \\
\text { Mental Health Policy Research; University of Toronto Faculty of Medicine, } \\
\text { Institute of Medical Science } \\
\text { Probst, Charlotte; Centre for Addiction and Mental Health, Institute for } \\
\text { Mental Health Policy Research; Technische Universitat Dresden, Institute of } \\
\text { Clinical Psychology and Psychotherapy \& Center of Clinical Epidemiology } \\
\text { and Longitudinal Studies } \\
\text { Gmel, Gerrit; Centre for Addiction and Mental Health, Institute for Mental } \\
\text { Health Policy Research; University of New South Wales, School of Electrical } \\
\text { Engineering and Telecommunications, Faculty of Engineering } \\
\text { Rehm, Jürgen; Centre for Addiction and Mental Health, Institute for Mental } \\
\text { Health Policy Research; University of Toronto, ; University of Toronto, } \\
\text { Dalla Lana School of Public Health; Technische Universitat Dresden, } \\
\text { Institute of Clinical Psychology and Psychotherapy \& Center of Clinical } \\
\text { Epidemiology and Longitudinal Studies }\end{array}$ \\
\hline $\begin{array}{r}\text { Is the invited manuscript for } \\
\text { consideration in a Special } \\
\text { Issue? : }\end{array}$ & Fetal Alcohol Spectrum Disorder \\
\hline Keyword: & $\begin{array}{l}\text { Alcohol use, Binge drinking, Fetal Alcohol Syndrome, Fetal Alcohol } \\
\text { Spectrum Disorder, Prevalence }\end{array}$ \\
\hline
\end{tabular}




\section{Mini Review}

\section{Global Prevalence of Alcohol Use and Binge Drinking During Pregnancy and Fetal Alcohol Spectrum Disorder}

\section{Svetlana Popova $^{1,2,3,4^{*}}$, Shannon Lange ${ }^{1,4}$, Charlotte Probst ${ }^{1,5}$, Gerrit Gmel ${ }^{1,6}$, \& Jürgen $\operatorname{Rehm}^{1,2,4,6,7}$}

${ }^{1}$ Institute for Mental Health Policy Research, Centre for Addiction and Mental Health, 33

Russell Street, Toronto, ON Canada M5S 2S1

${ }^{2}$ Dalla Lana School of Public Health, University of Toronto, 155 College Street, Toronto, ON Canada M5T 3M7

${ }^{3}$ Factor-Inwentash Faculty of Social Work, University of Toronto, 246 Bloor Street W., Toronto, ON Canada M5S 1V4

${ }^{4}$ Institute of Medical Science, University of Toronto, Faculty of Medicine, Medical Sciences Building, 1 King's College Circle, Toronto, ON Canada M5S 1A8

${ }^{5}$ Institute of Clinical Psychology and Psychotherapy \& Center of Clinical Epidemiology and Longitudinal Studies, Technische Universität Dresden, Chemnitzer Str. 46., 01187 Dresden, Germany

${ }^{6}$ School of Electrical Engineering and Telecommunications, Faculty of Engineering, The University of New South Wales, Sydney, NSW 2052, Australia

${ }^{7}$ Department of Psychiatry, University of Toronto, 250 College Street, Toronto, ON Canada M5T 1R8

\section{*Corresponding author:}

Svetlana Popova, Institute for Mental Health Policy Research, Centre for Addiction and Mental Health, 33 Russell Street, Toronto ON Canada M5S 2S1, e-mail: lana.popova@camh.ca, Tel: 1416-535-8501 ext. 34558 


\begin{abstract}
Alcohol use during pregnancy is an established cause of Fetal Alcohol Spectrum Disorder (FASD), with heavy drinking during pregnancy being explicitly linked to Fetal Alcohol Syndrome (FAS). The current paper presents recent estimates of the prevalence of: i) any amount of alcohol use during pregnancy, ii) one or more binge drinking episode(s) (four or more standard drinks on a single occasion) during pregnancy, iii) FAS, and iv) FASD among the general population globally and by World Health Organization region. It is apparent, based on the presented estimates, that alcohol use and binge drinking occur frequently among pregnant women in many countries and as a result, FASD is a prevalent alcohol-related developmental disability. Urgent action is required around the globe to eliminate prenatal alcohol exposure and prevent future children, adolescents and adults from having FASD.
\end{abstract}

Keywords: Alcohol use, Binge drinking, Fetal Alcohol Syndrome, Fetal Alcohol Spectrum Disorder, Prevalence 
In addition to being a risk factor for stillbirth (Kesmodel et al. 2002), spontaneous abortion (Henriksen et al. 2004), premature birth (Albertsen et al. 2004; Kemodel et al. 2000; Patra et al. 2011), intrauterine growth retardation (Patra et al. 2011; Yang et al. 2001) and low birth weight (O'Callaghan et al. 2003; Patra et al. 2011), alcohol use during pregnancy can interfere with the normal developmental progression of the fetus and result in the child being born with Fetal Alcohol Spectrum Disorder (FASD; Chudley et al. 2005; Hoyme et al. 2016). Despite this, a number of pregnancies are alcohol-exposed worldwide; it was estimated that globally $9.8 \%$ (95\% confidence interval $[\mathrm{CI}]: 8.9 \%-11.1 \%)$ of women consume alcohol while pregnant. In addition, it was estimated that the prevalence of alcohol use during pregnancy is the highest in the World Health Organization (WHO) European Region (EUR) at 25.2\% (95\% CI: $21.6 \%-29.6 \%)$ and the lowest in the Eastern-Mediterranean Region (EMR) at $0.2 \%(95 \% \mathrm{CI}$ : $0.1 \%-0.9 \%)$

With respect to the WHO regional estimates, according to the latest Global Status Report on Alcohol and Health 2014 (WHO 2014), almost all major alcohol indicators such as prevalence and level of consumption, rates of chronic and heavy episodic drinking, and alcohol use disorders are the highest in the EUR. Thus, it is understandable that this region has the highest prevalence of alcohol use during pregnancy, which was also found to exceed the global level. Further, regions that have the lowest prevalence of alcohol use while pregnant (EMR and South-East Asia Region [SEAR]) are also the regions where a large proportion of the population is abstainers (WHO 2014).

Given that the pattern, amount and/or critical period of prenatal alcohol exposure necessary for structural and/or functional teratogenesis are unknown, women are advised to completely abstain from alcohol during their entire pregnancy. However, among those regions 
where it was possible to estimate the prevalence of binge drinking (there were data limitations for the EMR and SEAR, which would undoubtedly have the lowest prevalence), it was estimated that the WHO region with the highest prevalence of binge drinking (four or more drinks on a single occasion) during pregnancy was the African Region (AFR) at 3.1\% (95\% CI: 0.1\%6.1\%), while the Western-Pacific Region (WPR) had the lowest prevalence at $1.8 \%$ (95\% CI: $0.0 \%-3.5 \%$; Table 1). Most alarmingly, the proportion of pregnant women that binge drank during pregnancy, among women who drank any amount of alcohol, was estimated to range from $10.7 \%$ in the EUR to $31.0 \%$ in the AFR (see Figure 1 for the prevalence of any amount of alcohol use and binge drinking during pregnancy and the estimated proportion of women who binge drank during pregnancy by WHO region and globally).

- Insert Table 1 about here -

- Insert Figure 1 about here -

The above estimates are extremely troublesome, as populations with a high prevalence of binge drinking have been found to have a high prevalence of FASD (May et al. 2007; Urban et al. 2008; Viljoen et al. 2005). The prevalence of Fetal Alcohol Syndrome (FAS) and FASD was estimated to be the highest in the EUR at 37.4 per 10,000 (95\% CI: 24.7-54.2 per 10,000) and 198.2 per 10,000 (95\% CI: 140.9-280.0 per 10,000), respectively, and the lowest in the EMR at 0.2 per 10,000 (95\% CI: $0.2-0.9$ per 10,000$)$ and 1.3 per 10,000 (95\% CI: $0.9-4.5$ per 10,000$)$, respectively. The global prevalence of FAS and FASD among the general population was estimated to be 14.6 per 10,000 (95\% CI: 9.4-21.7 per 10,000) and 77.3 (95\% CI: 49.0-116.1 per 10,000), respectively. Globally, the proportion of FAS cases among all FASD cases was 
$18.9 \%$ - that is, approximately two out of ten cases of FASD will have FAS (the most severe and visibly identifiable form of FASD). See Table 1 and Figure 2 for the prevalence of FAS and FASD by WHO region and globally.

- Insert Figure 2 about here -

We estimated the above reported prevalence of alcohol use during pregnancy and FASD using the current literature and advanced statistical methods to predict the prevalence wherever empirical data were unavailable. We systematically identified all available studies and performed country-specific random-effects meta-analyses to estimate the pooled prevalence for those countries with two or more empirical studies. For countries with one or no empirical studies, the prevalence of alcohol use and binge drinking during pregnancy was predicted using countrylevel indicators, while the prevalence of FAS and FASD was predicted based on the proportion of women who consumed alcohol during pregnancy that would give birth to a child with FAS and FASD, respectively. We then calculated a weighted average of the prevalence estimates, weighted by the number of live births in each country for the latest available year, in order to estimate the prevalence of alcohol use and binge drinking during pregnancy and FAS and FASD by WHO Region and globally. Lastly, we conducted Monte Carlo simulations in order to estimate the respective CI, by generating $1,000,000$ samples per country and using the $2.5^{\text {th }}$ and $97.5^{\text {th }}$ percentiles of the resulting distribution (Graham \& Talay 2013). For additional methodological details, see Popova and colleagues (2017). Importantly, in order to ensure consistency across studies and countries, the above estimations of alcohol use and binge drinking 
during pregnancy were restricted to self-report data, which has also been found to be a more conservative measure of alcohol use during pregnancy (Lange et al. 2014).

Although the timeframe covered in the literature searches were quite large (about 30 years), which was required in order to gather enough data for the analyses conducted, it was reasonable to expect that the prevalence of alcohol consumption during pregnancy has decreased over time. We did test for such a time-trend of decreasing prevalence of drinking during pregnancy in our prediction model and did not find a significant contribution, although there was a trend in this direction.

Prevalence estimates are vital for a number of notable reasons. Firstly, prevalence estimates provide an understanding of the scope of the problem and the population burden of disease. Secondly, prevalence estimates are needed in order to draw the attention of health care practitioners, public health authorities, policy makers and government officials to the public health problem at hand. Thirdly, prevalence estimates are necessary for setting priorities for public health policy, funding for public health initiatives and health care planning. Fourthly, prevalence estimates are essential to effectively prioritize, allocate resources for health care and prevention, and plan and deliver health care to high-needs populations such as women of childbearing age with alcohol use disorders, and individuals with FASD. Until now, in much of the world no prevalence estimates of alcohol use and binge drinking during pregnancy, and FAS and FASD exist and unfortunately, this may influence prioritization of health care expenditures for care related the populations most at-risk.

Overall, the figures presented above are alarming, and urgent action is required. While the above estimates apply to the general population, the prevalence was found to be much higher in special populations such as children in care, Aboriginal, correctional, psychiatric care, and low 
socioeconomic status populations, which were excluded from the study. Given that the current estimates were restricted to the general population, the findings emphasize that FASD is not restricted to disadvantaged groups, but rather occurs throughout society, regardless of socioeconomic status, education or ethnicity. Unfortunately, very few countries have empirical data on the prevalence of alcohol use and binge drinking during pregnancy and/or FAS and FASD, and for those that do, the majority of studies are outdated. Although the current estimates are a useful indicator of the public health burden of the condition and can provide a basis for health policy and resource allocation for FASD prevention initiatives, countries without data on the respective indicators are encouraged to conduct research to obtain their own prevalence data on alcohol use during pregnancy and FASD, and countries with outdated data are encouraged to conduct additional studies that are reflective of the current situation. Further, as an established public health problem, all countries should develop a surveillance system in order to monitor the prevalence of alcohol use during pregnancy and FASD over time. 


\section{References}

Albertsen, K., Andersen, A.N., Olsen, J., and Gronbaek, M. 2004. Alcohol consumption during pregnancy and the risk of preterm delivery. Am J Epidemiol. 159(2):155-161.

Chudley, A., Conry, J., Cook, J., Loock, C., Rosales, T., and LeBlanc, N. 2005. Fetal alcohol spectrum disorder: Canadian guidelines for diagnosis. CMAJ. 172(5 Suppl):S1-S21.

Graham, C., and Talay, D. Stochastic Simulation and Monte Carlo Methods: Mathematical Foundations of Stochastic Simulation. Berlin, Germany: Springer, 2013.

Henriksen, T.B., Hjollund, N.H., Jensen, T.K., Bonde, J.P., Andersson, A.M., Kolstad, H., et al. Alcohol consumption at the time of conception and spontaneous abortion. Am J Epidemiol. 160(7):661-667.

Hoyme, H.E., Kalberg, W.O., Elliott, A.J., Blankenship, J., Buckley, D., Marais, A.S., et al. Updated clinical guidelines for diagnosing fetal alcohol spectrum disorders. Pediatrics. 2016. 138(2):e20154256.

Kesmodel, U., Olsen, S.F., and Secher, N.J. Does alcohol increase the risk of preterm delivery? Epidemiology. 2000. 11(5):512-518.

Kesmodel, U., Wisborg, K., Olsen, S.F., Henriksen, T.B., and Secher, N.J. Moderate alcohol intake during pregnancy and the risk of stillbirth and death in the first year of life. Am J Epidemiol. 2002. 155(4):305-312.

Lange, S., Shield, K., Koren, G., Rehm, J., and Popova, S. A comparison of the prevalence of prenatal alcohol exposure obtained via maternal self-reports versus meconium testing: a systematic literature review and meta-analysis. BMC Pregnancy Childbirth. 2014.

$14: 127$. 
May, P.A., Gossage, J.P., Marais, A.S., Adnams, C.M., Hoyme, H.E., Jones, K.L., et al. The epidemiology of fetal alcohol syndrome and partial FAS in a South African community. Drug Alcohol Depend. 2007. 88(2-3):259-271.

O'Callaghan, F.V., O'Callaghan, M., Najman, J.M., Williams, G.M., and Bor, W. Maternal alcohol consumption during pregnancy and physical outcomes up to 5 years of age: a longitudinal study. Early Hum Dev. 2003. 71(2):137-148.

Patra, J., Bakker, R., Irving, H., Jaddoe, V.W.V., Malini, S., and Rehm, J. Dose-response relationship between alcohol consumption before and during pregnancy and risk of low birth weight, preterm birth and small for gestational age (SGA) - A systematic review and meta-analyses. BJOG. 2011. 118(12):1411-1421.

Popova, S., Lange, S., Probst, C., Gmel, G., and Rehm, J. Estimation of national, regional, and global prevalence of alcohol use during pregnancy and fetal alcohol syndrome: a systematic review and meta-analysis. Lancet Glob Health. 2017. 5(3):e290-e299.

Urban, M., Chersich, M.F., Fourie, L.A., Chetty, C., Olivier, L., and Viljoen, D. Fetal alcohol syndrome among grade 1 schoolchildren in Northern Cape Province: prevalence and risk factors. S Afr Med J. 2008. 98(11):877-882.

Viljoen, D.L., Gossage, J.P., Brooke, L., Adnams, C.M., Jones, K.L., Robinson, L.K., et al. Fetal alcohol syndrome epidemiology in a South African community: A second study of a very high prevalence area. J Stud Alcohol. 2005. 66(5):593-604.

World Health Organization (WHO). 2014. Global status report on alcohol and health 2014. Geneva, Switzerland: WHO. [Available from http://apps.who.int/iris/bitstream/10665/112736/1/9789240692763_eng.pdf] 
Yang, Q., Witkiewicz, B.B., Olney, R.S., Liu, Y., Davis, M., Khoury, M.J., et al. A case-control study of maternal alcohol consumption and intrauterine growth retardation. Ann Epidemiol. 2001. 11(7):497-503. 
Table 1. Global prevalence of any amount of alcohol use and binge drinking (4 or more drinks on a single occasion) during pregnancy, and FAS and FASD among the general population in 2012, by WHO region

\begin{tabular}{|c|c|c|c|c|c|c|c|c|c|c|c|c|}
\hline \multirow[t]{3}{*}{$\begin{array}{l}\text { WHO } \\
\text { region }\end{array}$} & \multicolumn{3}{|c|}{$\begin{array}{l}\text { Alcohol use (any amount) } \\
\text { during pregnancy }(\%)^{\mathrm{a}}\end{array}$} & \multicolumn{3}{|c|}{$\begin{array}{c}\text { Binge drinking during } \\
\text { pregnancy }(\%)^{b}\end{array}$} & \multicolumn{3}{|c|}{ FAS (per 10,000) } & \multicolumn{3}{|c|}{ FASD $($ per 10,000) } \\
\hline & \multirow[t]{2}{*}{$\mathbf{P E}$} & \multicolumn{2}{|c|}{$95 \%$ CI } & \multirow[t]{2}{*}{$\mathbf{P E}$} & \multicolumn{2}{|c|}{$95 \%$ CI } & \multirow[t]{2}{*}{$\mathbf{P E}$} & \multicolumn{2}{|c|}{$95 \%$ CI } & \multirow[t]{2}{*}{$\mathbf{P E}$} & \multicolumn{2}{|c|}{$95 \% \mathrm{CI}$} \\
\hline & & Lower & Upper & & Lower & Upper & & Lower & Upper & & Lower & Upper \\
\hline AFR & 10.0 & 8.5 & 11.8 & 3.1 & 0.1 & 6.1 & 14.8 & 8.9 & 21.5 & 78.3 & 53.6 & 107.1 \\
\hline AMR & 11.2 & 9.4 & 12.6 & 2.8 & 0.1 & 5.6 & 16.6 & 11.0 & 24.0 & 87.9 & 63.7 & 132.4 \\
\hline EMR & 0.2 & 0.1 & 0.9 & - & - & - & 0.2 & 0.2 & 0.9 & 1.3 & 0.9 & 4.5 \\
\hline EUR & 25.2 & 21.6 & 29.6 & 2.7 & 0.0 & 5.3 & 37.4 & 24.7 & 54.2 & 198.2 & 140.9 & 280.0 \\
\hline SEAR & 1.8 & 0.9 & 5.1 & - & - & - & 2.7 & 1.3 & 8.1 & 14.1 & 6.4 & 53.1 \\
\hline WPR & 8.6 & 4.5 & 11.6 & 1.8 & 0.0 & 3.5 & 12.7 & 7.7 & 19.4 & 67.4 & 45.4 & 116.6 \\
\hline Global & 9.8 & 8.9 & 11.1 & - & - & - & 14.6 & 9.4 & 23.3 & 77.3 & 49.0 & 116.1 \\
\hline
\end{tabular}

${ }^{\mathrm{a}}$ The prevalence of any amount of alcohol use during pregnancy is inclusive of the prevalence of binge drinking during pregnancy

${ }^{\mathrm{b}}$ It was not possible to estimate the prevalence of binge drinking during pregnancy for the Eastern-Mediterranean Region and South-

East Asia Region due to a lack of available data for countries in these regions and therefore, the global prevalence could not be estimated

${ }^{\mathrm{c}}$ The prevalence of FASD is inclusive of the prevalence of FAS

$\mathrm{AFR}=\mathrm{A}$ frican Region, $\mathrm{AMR}=$ Region of the Americas, $\mathrm{CI}=$ Confidence Interval, $\mathrm{EMR}=$ Eastern-Mediterranean Region,

EUR=European Region, FAS=Fetal Alcohol Syndrome, FAS=Fetal Alcohol Spectrum Disorder; PE=Prevalence Estimate,

$\mathrm{SEAR}=$ South-East Asia Region, $\mathrm{WHO}=$ World Health Organization, WPR=Western Pacific Region 


\section{Figure Captions}

Figure 1. The prevalence (\%) of any amount of alcohol use ${ }^{\mathrm{a}}$ and binge drinking during pregnancy and the estimated proportion of women who binge drank during pregnancy (percent shown on the top of bars) by World Health Organization region $\mathrm{AFR}=\mathrm{African}$ Region, $\mathrm{AMR}=$ Region of the Americas, $\mathrm{EMR}=$ Eastern-Mediterranean Region, EUR=European Region, SEAR=South-East Asia Region, WPR=Western Pacific Region

${ }^{\mathrm{a}}$ The prevalence of any amount of alcohol use during pregnancy is inclusive of the prevalence of binge drinking during pregnancy

Note. It was not possible to estimate the prevalence of binge drinking during pregnancy for the EMR and SEAR due to a lack of available data for countries in these regions and therefore, the global prevalence could not be estimated

Figure 2. The prevalence (per 10,000) of FASD ${ }^{\mathrm{a}}$ and FAS (in brackets) by World Health Organization region

$\mathrm{AFR}=\mathrm{African}$ Region, $\mathrm{AMR}=$ Region of the Americas, $\mathrm{EMR}=$ Eastern-Mediterranean Region, EUR=European Region, SEAR=South-East Asia Region, WPR=Western Pacific Region

${ }^{a}$ The prevalence of FASD is inclusive of the prevalence of FAS 


$\begin{array}{lllll}\text { WHO region } & \text { Binge drinking (Alcohol use (any amount) } & \text { Alcohol use } & \\ \text { AFR } & 3.1 & 6.9 & 10 & 31.0 \% \\ \text { AMR } & 2.8 & 8.4 & 11.2 & 25.0 \% \\ \text { EMR } & & 0.2 & 0.2 & \\ \text { EUR } & 2.7 & 22.5 & 25.2 & 10.7 \% \\ \text { SEAR } & & 1.8 & 1.8 & \\ \text { WPR } & 1.8 & 6.8 & 8.6 & 20.9 \% \\ \text { Global } & & 9.8 & 9.8 & \end{array}$




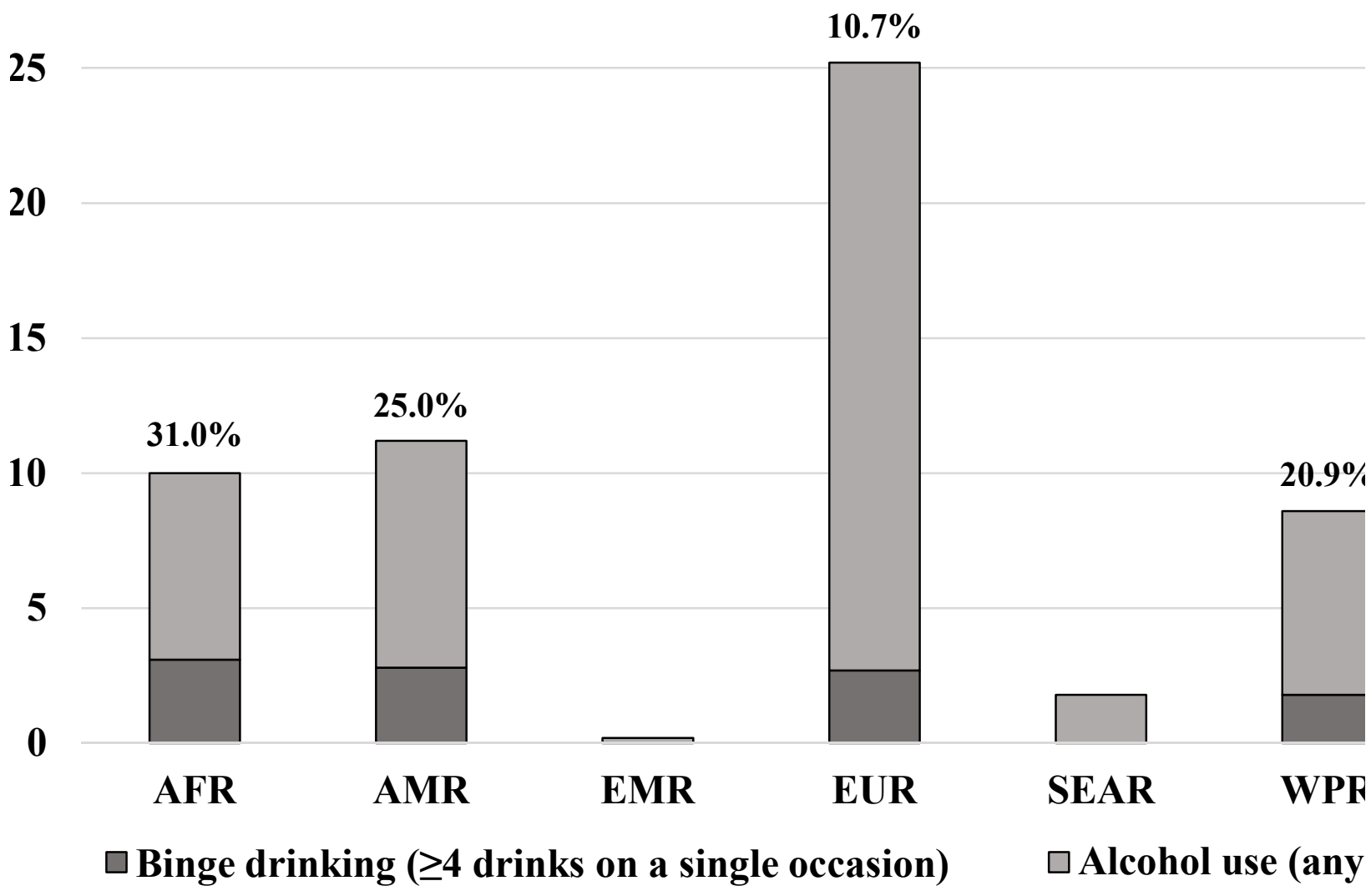




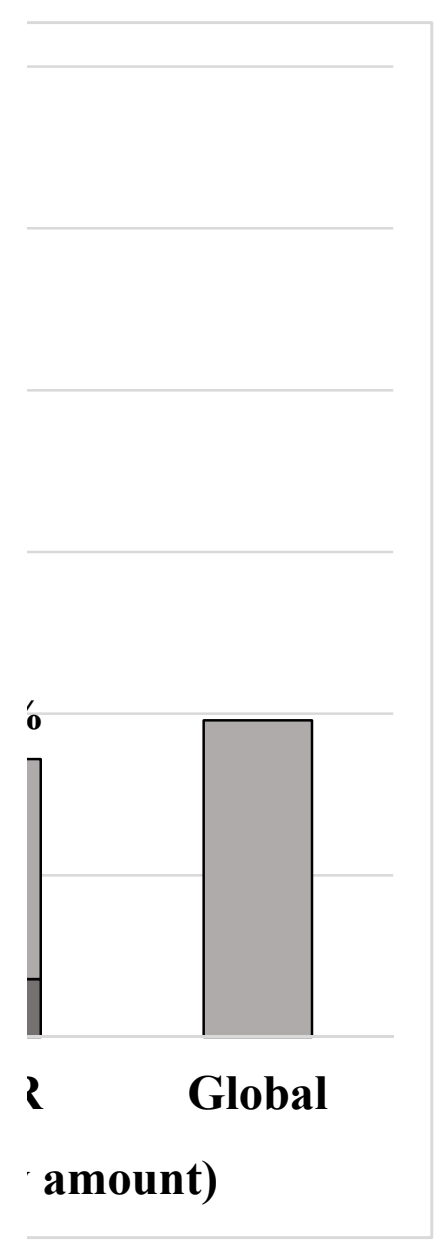

https://mc06.manuscriptcentral.com/bcb-pubs 
WHO region FAS

AFR

AMR

EMR

EUR

SEAR

WPR

Global
14.8

16.6

0.2

37.4

2.7

12.7

14.6
FASD

63.5

71.3

1.1

160.8

11.4

54.7

62.7
FASD

78.3

87.9

1.3

198.2

14.1

67.4

77.3

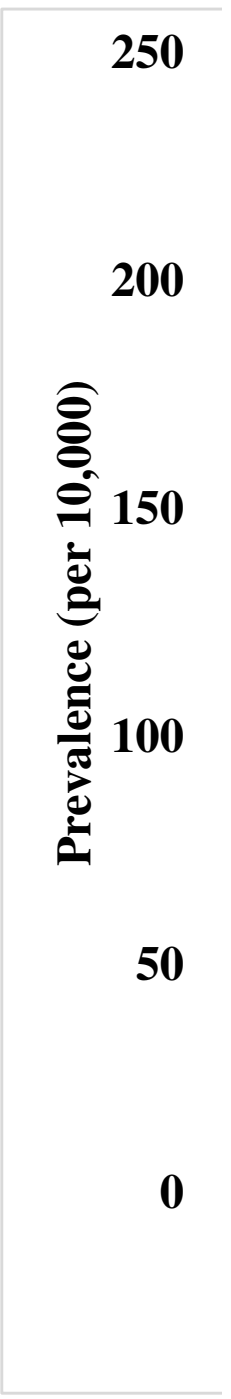


[VALUE] (37.4)

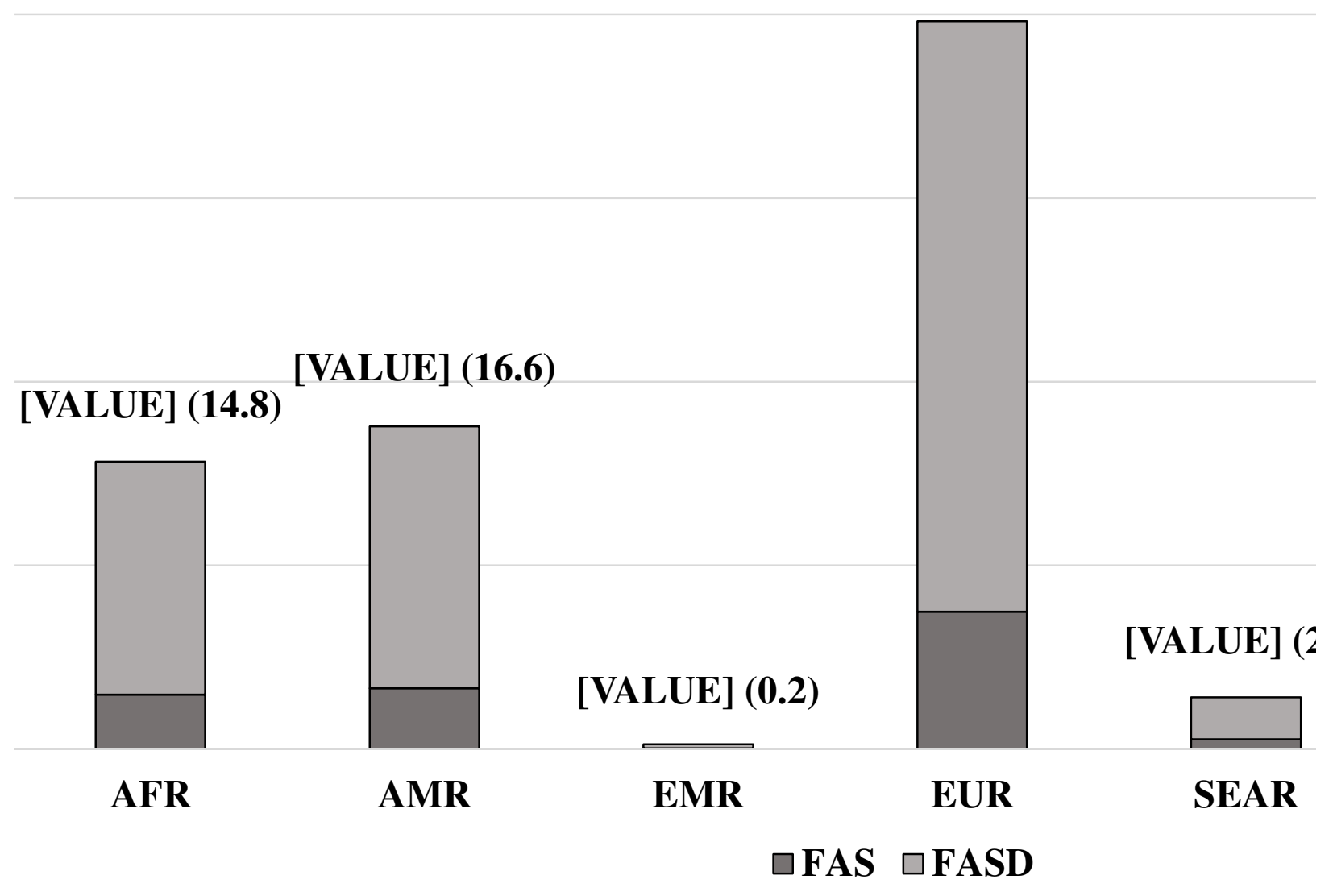




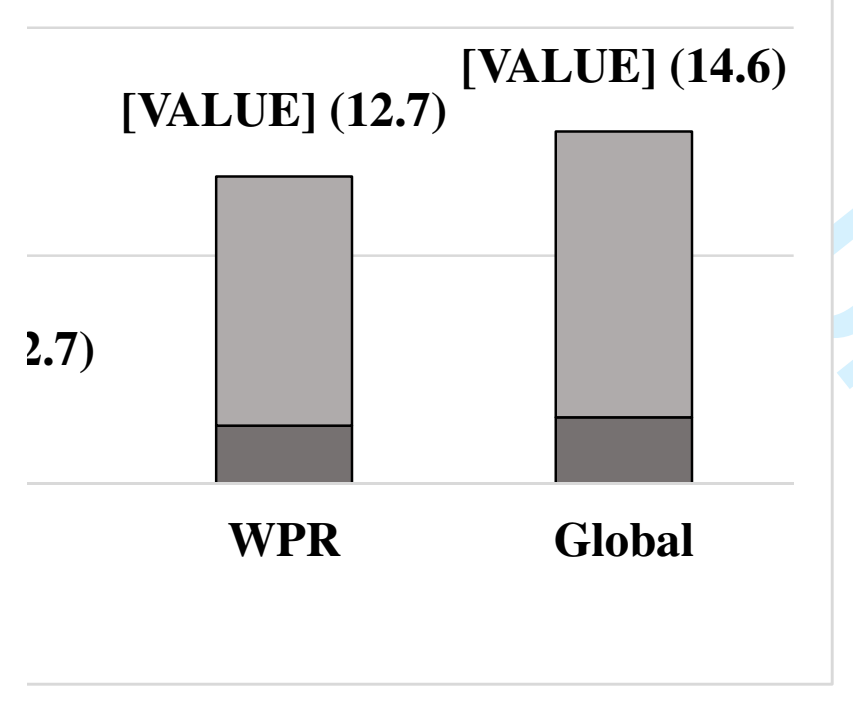

